\title{
Carbon Nanotube Foils for Electron Stripping in Tandem Accelerators
}

\author{
Karl von Reden ${ }^{\mathrm{a}}$, Mei Zhang ${ }^{\mathrm{b}}$, Martha Meigs ${ }^{\mathrm{c}}$, Enid Sichel ${ }^{\mathrm{a}}$, Shaoli Fang ${ }^{\mathrm{b}}$, and Ray H. \\ Baughman $^{\mathrm{b}}$ \\ a. Woods Hole Oceanographic Institution, Woods Hole, MA 02543 \\ b. NanoTech Institute, UT Dallas, Richardson, TX 75083 \\ c. Oak Ridge National Laboratory, HRIBF, Oak Ridge, TN 37831
}

\begin{abstract}
Carbon nanotube technology has rapidly advanced in recent years, making it possible to create meterlong, $\sim 4 \mathrm{~cm}$ wide films of multi-walled tubes of less than $3 \mu \mathrm{g} / \mathrm{cm}^{2}$ areal density in a bench top open-air procedure [1]. The physical properties of individual carbon nanotubes have been well established, equaling or surpassing electrical and thermal conductivity and mechanical strength of most other materials, graphite in particular. The handling and transport of such nanotube films, dry-mounted self-supporting on metal frames with several $\mathrm{cm}^{2}$ of open area, is problem-free: the aerogel films having a volumetric density of about $1.5 \mathrm{mg} / \mathrm{cm}^{3}$ survived the trip by car and air from Dallas to Oak Ridge without blemish. In this paper we will present the results of first tests of these nanotube films as electron stripper media in a tandem accelerator. The tests were performed in the Model 25 URC tandem [2] of the Holifield Radioactive Ion Beam Facility (HRIBF) at Oak Ridge National Laboratory. We will discuss the performance of nanotube films in comparison with chemical vapor deposition and laser-ablated carbon foils.
\end{abstract}

PACS codes: 81.07.-b; 73.50.-h; 29.27.-a; 34.70.+e. Keywords: nanoscale materials; transport phenomena; thin films; charged particle beams; charge transfer.

\section{Introduction}

Tandem accelerators are equipped with terminal stripper devices to remove two or more electrons from the incoming negative ions and end up with an accelerated positive ion beam of $(\mathrm{q}+1) \mathrm{V}_{\mathrm{T}}$ kinetic energy, where $\mathrm{q}$ is the final charge state and $\mathrm{V}_{\mathrm{T}}$ is the terminal voltage of the accelerator. The advantage of the now common gas stripping over the traditional method of foil stripping is quite obvious: gas can be continually replenished and the thickness of the stripping medium can be adjusted during operation to achieve optimal efficiency for a given (q, $\mathrm{V}_{\mathrm{T}}$ ) setup, whereas foils are usually fragile, have to be carefully chosen to have the correct thickness for a given application, have a limited lifetime and may have non-linear aging properties. However, unless quite elaborate terminal vacuum pumping is applied, gas stripper devices lead to elevated pressure in the accelerator tubes with beam quality degradation and possibly reduced transmission due to repeated charge exchange and straggling. These beam degrading effects become more important at lower energies $\left(\mathrm{V}_{\mathrm{T}}<1 \mathrm{MV}\right)$, an energy regime that allows building more compact systems, for example for Accelerator Mass Spectrometry (AMS). Beams emerging from such low-energy accelerators with gas strippers can be quite large in divergence and therefore require large-gap analyzing magnets and electrostatic analyzers, making a compact design difficult to achieve. It is therefore desirable to use solid state stripper media in compact systems. For this to work, thin foils have to be developed that are easy to handle, are at least as durable under irradiation, and have a charge exchange efficiency comparable with the graphite foils presently in use. Earlier investigations with conventional foils have shown that AMS would benefit from this development [3].

*Corresponding author: kvonreden@whoi.edu, Woods Hole Oceanographic Institution, MS 8, Woods Hole, MA 02543, Phone: 508-289-3384, Fax: 508-457-2183 


\section{Conventional stripper foil technology}

Most accelerator facilities use thin carbon foils as the stripper medium if gas stripping is not an option. However, for areal densities less than $10 \mu \mathrm{g} / \mathrm{cm}^{2}$, the task of creating the actual stripper foils is a tedious multi-step process. The foils are deposited in vacuum on a substrate by one of several methods (chemical vapor deposition, laser ablation, arc discharge). Separation from the substrate and purification may require strong solvents in which the foils are floated free of the substrate and caught on the foil holder frames. After removal from the substrate the foils are very fragile and will most likely not survive extended transportation or even casual handling. Depending on the application and type of foil, the lifetime of a conventional stripper foil under ion beam irradiation can be from minutes to a few days. The method does not lend itself to an installation where the accelerator is operated as an application tool in an interdisciplinary laboratory environment like an accelerator mass spectrometry facility.

\section{Search for alternatives}

Recent progress in the field of nano technology has introduced the possibility of creating strong films of entangled nanotubes to use as stripper foils. The initial difficulty has been to make films thin enough to be of use in our application. Maximum length of individual nanotube bundles originally was only of the order of a few $\mu \mathrm{m}$, such that mats of entangled tubes needed to be more than $100 \mathrm{~nm}$ thick to be self-supporting [4]. For a realistic stripping test these mats were too thick. Last year's development [1] of a simple method to create large area $50 \mathrm{~nm}$ thick highly aligned nanotube films from a "forest" of more than $200 \mu \mathrm{m}$ long multi-walled nanotube bundles, grown on a substrate, gave us the ability to perform a test in the terminal of a tandem accelerator. Such thin multiwalled carbon nanotube films are obtained by densification of much thicker aerogel films, having a density of only about $0.0015 \mathrm{~g} / \mathrm{cm}^{3}$, and these aerogel films were used in the present study. The areal density of both the aerogel films and the densified aerogel films is the same, about $3 \mu \mathrm{g} / \mathrm{cm}^{3}$. This areal density is for a single film layer, and the present study is for both single film layers and stacked film layers bonded together by van der Waals attraction. The major advantage of these films is their ease of handling and transport. The structure of the films is parallel aligned strands of entangled nanotube bundles. Normal handling in air is unproblematic. Properly packaged, the free-standing films can even be mailed. Mounting the films on foil holders is easily accomplished in air by allowing the as-drawn aerogel film to come in contact with the metal frame and shearing off the excess around the outside of the frame. No further treatment is necessary.

\section{Electrical properties of the films}

Individual single walled carbon nanotubes are known to be either semi-conducting or conducting, depending on the geometric orientation of bonds within the bundles. The presently investigated multiwalled nanotubes, which comprise concentrically arrayed single walled carbon nanotubes, are metallic. Even though the films in this study are aligned strands of nanotube bundles, scanning electron microscopy and atomic force microscopy images indicate that these bundles fork and then recombine with forks from other nanotube bundles to provide a degree of 
lateral connectivity. It is therefore of interest to measure the electrical conductivity of a film to estimate its ability to carry away the charge deposited by the ion beam during the stripping process. Using the Keithley Delta Mode system [5] (6221 DC/AC Current source and 2182A Nanovoltmeter), measurements were made on a $2.2 \mathrm{~mm}$ wide, $3.64 \mathrm{~mm}$ long sample section of the material, pictured in figure 1a. The width was chosen to match roughly the size of the core of an ion beam after acceleration towards a tandem terminal. Figure $1 \mathrm{~b}$ shows the response of the film to a stream of injected $500 \mu$ s square current pulses ranging from $-80 \mathrm{~mA}$ to $+80 \mathrm{~mA}$ amplitude. Voltage measurements were taken $250 \mu$ s after pulse start and the duty cycle was 80 ms. The measured resistance ranged from $0.87 \mathrm{k} \Omega$ at high current to $1.5 \mathrm{k} \Omega$ at low current. Using the reported areal density of the films $\left(2.7 \mu \mathrm{g} / \mathrm{cm}^{2}\right)$, the estimated resistivity of the material $(6 \mathrm{x}$ $10^{-6}-1 \times 10^{-5} \Omega \mathrm{m}$ ) is sufficiently low to serve its purpose in this application. The nonlinearity and slight asymmetry in the V(I) and R(I) curves could be thermal effects.

\section{Stripper foil test at HRIBF (ORNL)}

A set of ten as-drawn nanotube films were mounted on HRIBF stripper foil holders at the Nanotech Institute of UT Dallas. Figure 2 shows the mounting characteristics of the foils. The double and triple layer foils are not the ideal method to increase the thickness as the nanotube bundle strands from different layers will not perfectly line up to fill the gaps in the case of the parallel orientation and thick cross points will be present in the case of crossed strands. Other methods of areal densification are under development. After preparation at the NanoTech Institute the foil holders were hand-carried to ORNL and mounted in the stripper foil ladder of the tandem accelerator together with a set of conventional graphite foils. The test was designed to cover a broad range of ion energies and masses, comparing the performance of the nanotube foils with that of chemical vapor deposition (CVD) and laser-ablation graphite foils. ${ }^{58} \mathrm{Ni}$ at $\sim 20$ MV terminal voltage is a well understood beam at HRIBF and was the choice for a starting point. For AMS in smaller accelerators a lower energy ${ }^{12} \mathrm{C}$ beam is of interest. $2.5 \mathrm{MV}$ terminal voltage was chosen to match the parameters of the NOSAMS [6] system in Woods Hole. As an endurance test, ${ }^{197} \mathrm{Au}$ at $20 \mathrm{MV}$ terminal voltage is known to challenge any solid state stripping device. Finally, ${ }^{1} \mathrm{H}$ at $20 \mathrm{MV}$ terminal voltage was used to test foil/gas stripping in combination. Beam currents were measured in Faraday cups (FC) at three locations in the system: injection point (FC 13-1), terminal exit (FC T-2), and after the high-energy analyzing magnet (FC 17-2).

\section{Results}

Using ${ }^{58} \mathrm{Ni}$ as the injected beam we performed charge state scans for several of the nanotube and graphite foils to establish a baseline for the test. Figure 3 shows the results for four multi-walled nanotube (MWNT) films in comparison with a $4 \mu \mathrm{g} / \mathrm{cm}^{2}$ laser ablation graphite foil. The average areal density of the single layer MWNT foil is $2.7 \mu \mathrm{g} / \mathrm{cm}^{2}$ (68\% of the graphite foil). The measured current at charge state $12^{+}$is $9.3 \mathrm{pnA}$ for the MWNT foil and $15.4 \mathrm{pnA}$ for the graphite foil (60\%). In light of the fact that the area coverage of the nanotube bundle strands is significantly less than $100 \%$, this result is encouraging. As mentioned above, increasing the thickness by double and triple layering does not raise the stripping target area coverage proportionally. In fact, as can be seen in the crossed layer case, we now have introduced blind 
spots of excessive thickness in addition to the remaining holes between the crossed strands, in effect lowering the charge exchange efficiency by over $40 \%$ compared to the single-layer foil and moving the optimal charge state to a lower value. To assess the quality of the positive ion beam after charge exchange, current measurements were also taken at FC 17-2 for some of the foils at the optimal charge state. The transmission between FC T-2 and FC 17-2 was 64\% for the laser ablation graphite foil, 73\% for the single layer MWNT foil (\#4), 56\% for the double layer MWNT foil (\#7), and 65\% for the triple layer MWNT foil (\#6). This result suggests a higher beam quality (less divergence) for the single layer MWNT foil than for any of the others.

The next test involved ${ }^{12} \mathrm{C}$ at a terminal voltage of $2.5 \mathrm{MV}$. The charge exchange efficiency (to the optimal $3^{+}$charge state) of single layer MWNT foil \#1 was found to be $55 \%$ lower than that of a $4 \mu \mathrm{g} / \mathrm{cm}^{2}$ graphite foil for this beam and energy, but the transmission between FC T-2 and FC $17-2$ was $45 \%$ higher with the MWNT foil, bringing the net efficiency to $80 \%$. The main goal of this test series was, however, to determine the long exposure time performance of a MWNT foil. Figure 4 shows the measured beam currents for MWNT foil \#1 in an overnight run. While there appears to be some initial conditioning effect, the foil held up without being moved laterally (rotated), tracking the slowly rising injected beam current overnight without indication of impending failure.

The true test of the durability of the MWNT foils had to be done with heavy ions, known to quickly destroy conventional foils. For this test ${ }^{197} \mathrm{Au}$ and a terminal voltage of $\sim 20 \mathrm{MV}$ were used. Figure 5 shows the performance of several foils under bombardment with the heavy ions. None of the foils could sustain the abuse on one spot for more than 0.5 hours. The HRIBF stripper foil actuator allows moving the holders to find "sweet spots" on a foil. This rotating motion can be performed several times to obtain lifetimes of several hours for a good foil. All MWNT foils and the CVD foils displayed immediate degradation in stripping efficiency after start of the exposure at any given spot. Only the laser ablation graphite foil was stable for $15-20$ minutes before succumbing to damage from the gold ions.

A final test was performed to evaluate the effect of adding a small amount of stripper gas to a MWNT foil. This test was done with protons at $20 \mathrm{MV}$ terminal voltage. Using $\mathrm{N}_{2}$ as stripper gas, the flow was adjusted such that the beam line pressure rose from $1.4 \times 10^{-8}$ mbar to $2.1 \times 10^{-8}$ mbar. This corresponded with a rise in transmission efficiency by $6.3 \%$ for MWNT foil \#1, not enough to make this approach viable.

\section{Discussion}

MWNT stripper foils of the type described here are capable of performing at least at the level of conventional CVD carbon foils. Their disadvantage (at this point) is the insufficient area coverage of the nanotube bundle strands, allowing only part of the foil area to contribute to the stripping process. The observed stripping efficiencies for the various MWNT foils are in line with expectations. Increasing the area coverage has to be the main goal of further development of this technology. Simply doubling or tripling the layer thickness on a frame (with parallel or crossed nanotube orientation directions) does not accomplish this. The NanoTech group has already developed a densification method that leads to thinner stronger films, possibly better 
suited for this application. One other possibility that could not be investigated in this study is the use of foil holders with individually rotatable and removable multiple frames to find the optimal combination for a given ion beam. The clear advantage of MWNT foils is the simplicity of their assembly (assuming the availability of a substrate-grown forest of nanotubes) and the ease of handling them afterwards. The nanotube structure appears to hold up well under light to medium heavy ion bombardment but does not seem to be well suited for heavy ion applications. For the latter, the tremendous force of a single ion hit on a nanotube bundle appears to lead to its immediate disintegration and probable damage to neighboring bundles as well. This assumption will be further researched by post-irradiation inspection with high resolution imaging when the foils will be retrieved from the HRIBF tandem, in the fall of this year.

\section{Acknowledgments}

This work was supported by a grant from the "Cecil H. and Ida M. Green Technology Innovation Awards" program of the Woods Hole Oceanographic Institution and in part by the U.S. National Science Foundation through Cooperative Agreement 82899613 and the Robert A. Welch Foundation grant AT-0029. We thank Frank Hennrich from the Forschungszentrum Karlsruhe for supplying us with his nanotube films for our initial research.

\section{References}

[1] M. Zhang, S. Fang, A. Zakhidov, S. Lee, A. Aliev, C. Williams, K. Atkinson, R. Baughman, SCIENCE 309, 8/19/2005, 1215-1219.

[2] Manufactured by National Electrostatics Corporation, Middleton, WI 53562, USA

[3] B. Hughey, R. Shefer, R. Klinkowstein, X. Zhao, W. Kieser, A. Litherland, Nucl. Inst. Meth. B123 (1997) $186-192$.

[4] F. Hennrich, S. Lebedkin, S. Malik, J. Tracy, M. Barczewski, H. Rösner, M. Kappes, Phys. Chem. Chem. Phys. 4 (2002) 2273 - 2277.

[5] Keithley Instruments Inc, Cleveland, Ohio 44139, USA

[6] National Ocean Sciences Accelerator Mass Spectrometry Facility, Woods Hole Oceanographic Institution - http://www.nosams.whoi.edu/about/index.html 


\section{Figure captions}

Figure 1a: Single-layer multi-walled nanotube film spanning two $0.8 \mathrm{~mm}$ diameter wire electrodes for electric conductivity measurements. The image is shown as negative to better highlight the strand structure of the film.

Figure 1b: V(I) and R(I) curves for the nanotube film shown in fig. 1a. The "asymmetry" curves in the positive sector are the R(-I) and $-\mathrm{V}(-\mathrm{I})$ data to show the small difference between the polarities. See text for further discussion.

Figure 2: MWNT foils from the NanoTech Institute (UT Dallas) mounted on HRIBF stripper foil holders with 0.75 inch diameter circular opening. Description: \#1 - \#4 are single layer foils as shown in figure 1a; \#5 and \#6 are triple layer foils with parallel orientation of the nanotube bundle strands; \#7 is a double layer foil with parallel orientation; \#8 and \#9 are crossed double layer foils; \#10 is a triple layer foil not used in this study.

Figure 3: Final charge state scans for five of the stripper foils showing $12^{+}$as the optimal value for both the graphite foil and the MWNT foils, with a shift to a lower value for the crossed double layer foil.

Figure 4: Overnight test of single layer MWNT foil \#1 with a $2.5 \mathrm{MeV}{ }^{12} \mathrm{C}$ beam. The injected beam current (FC 13-1) is represented by black diamonds. The analyzed beam (black circles) at FC 17-2 reflects charge state $3^{+}$.

Figure 5: Endurance test with a $20 \mathrm{Mev}^{197} \mathrm{Au}^{-}$beam. Notice the logarithmic scale on the ordinate, better matching the range of the data. At times marked by " $\mathrm{R}$ " the foil under bombardment was moved to find an undamaged area. 


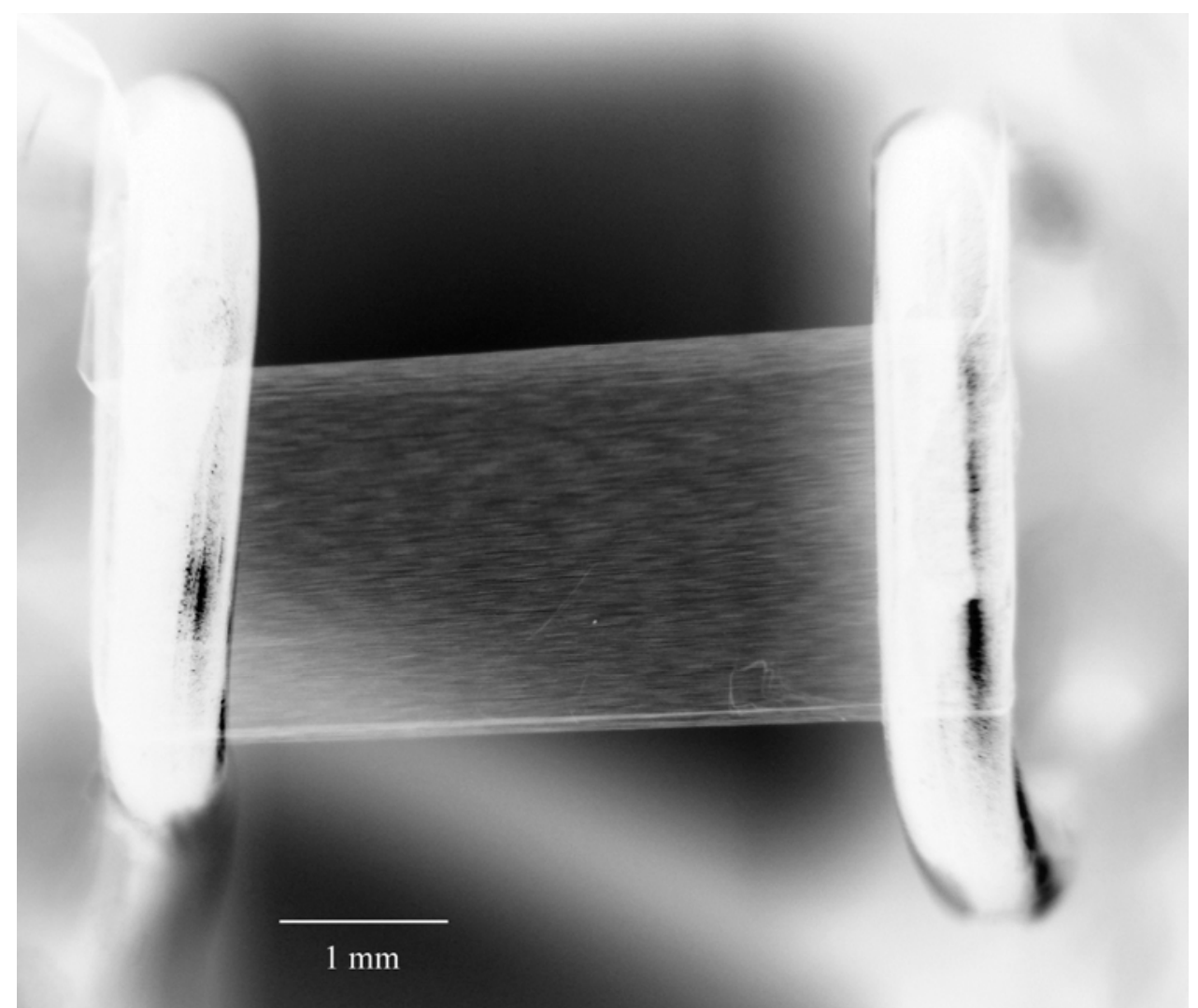

Figure 1a

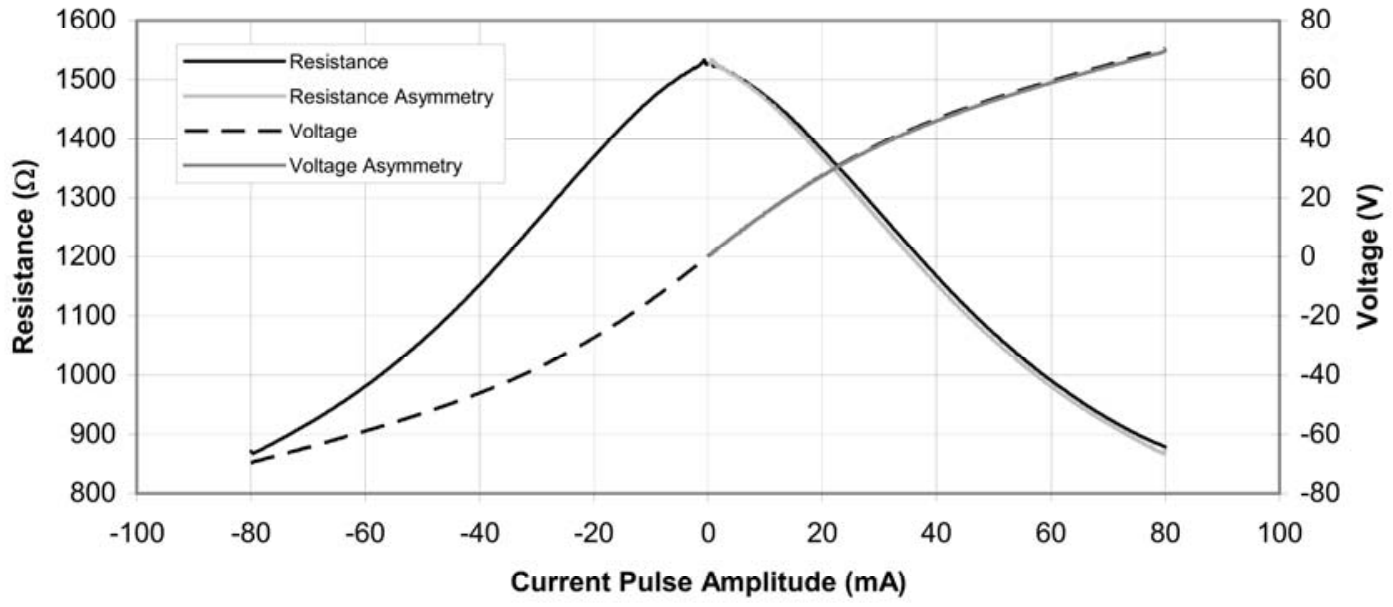

Figure 1b 


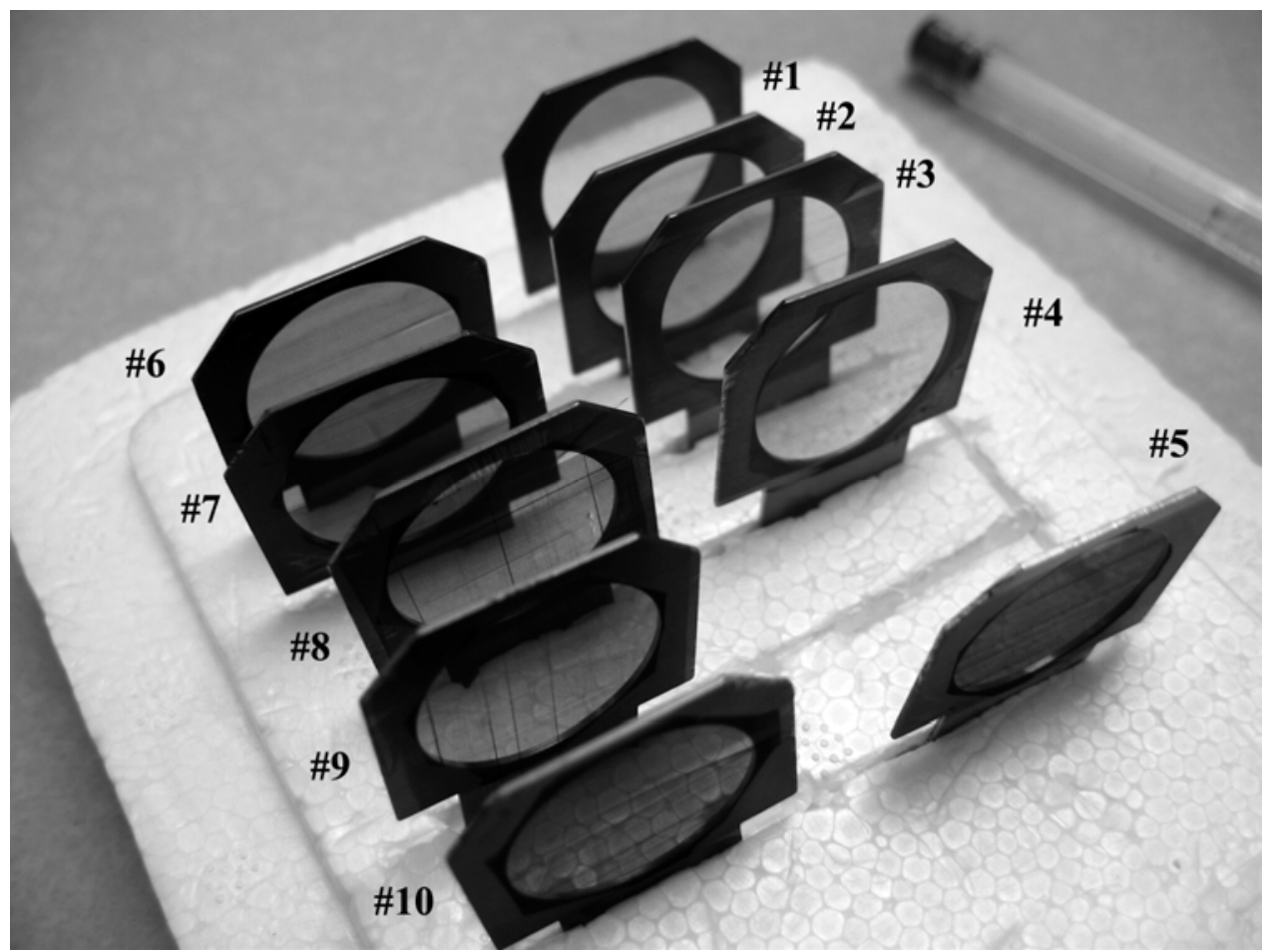

Figure 2

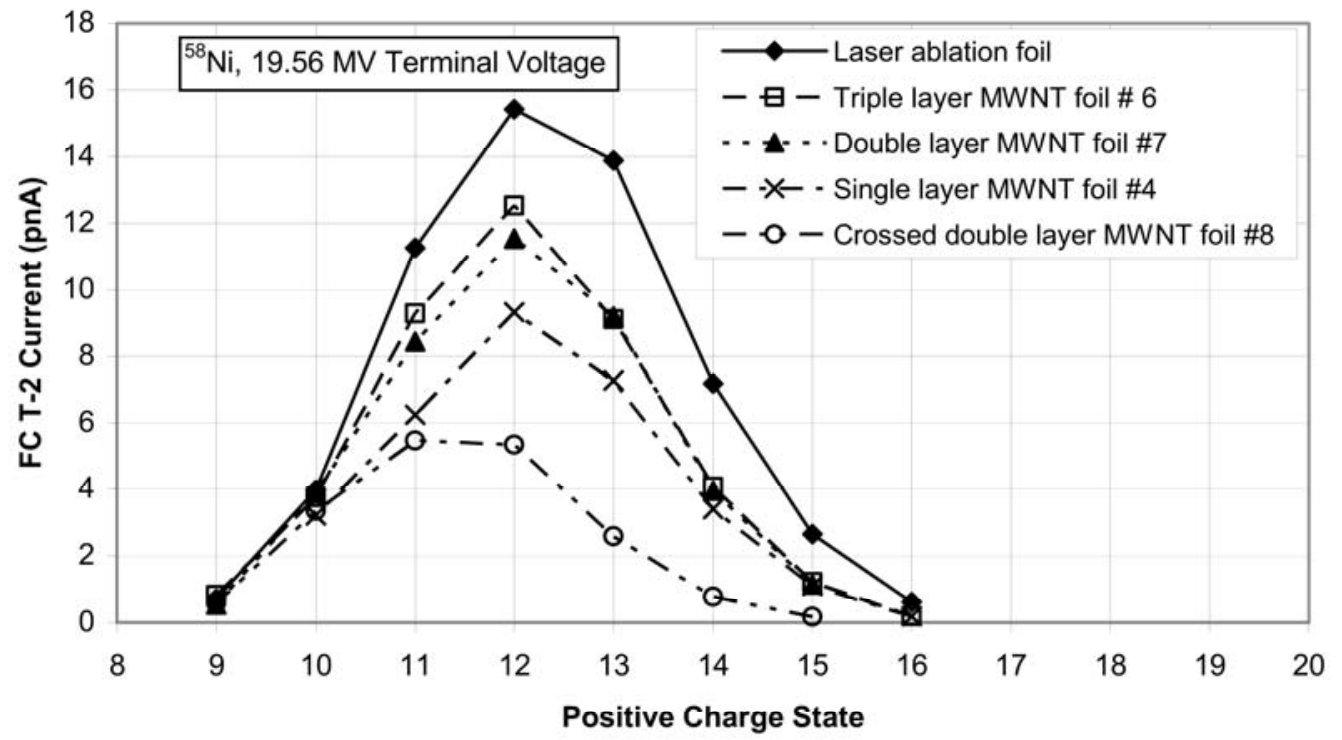

Figure 3 


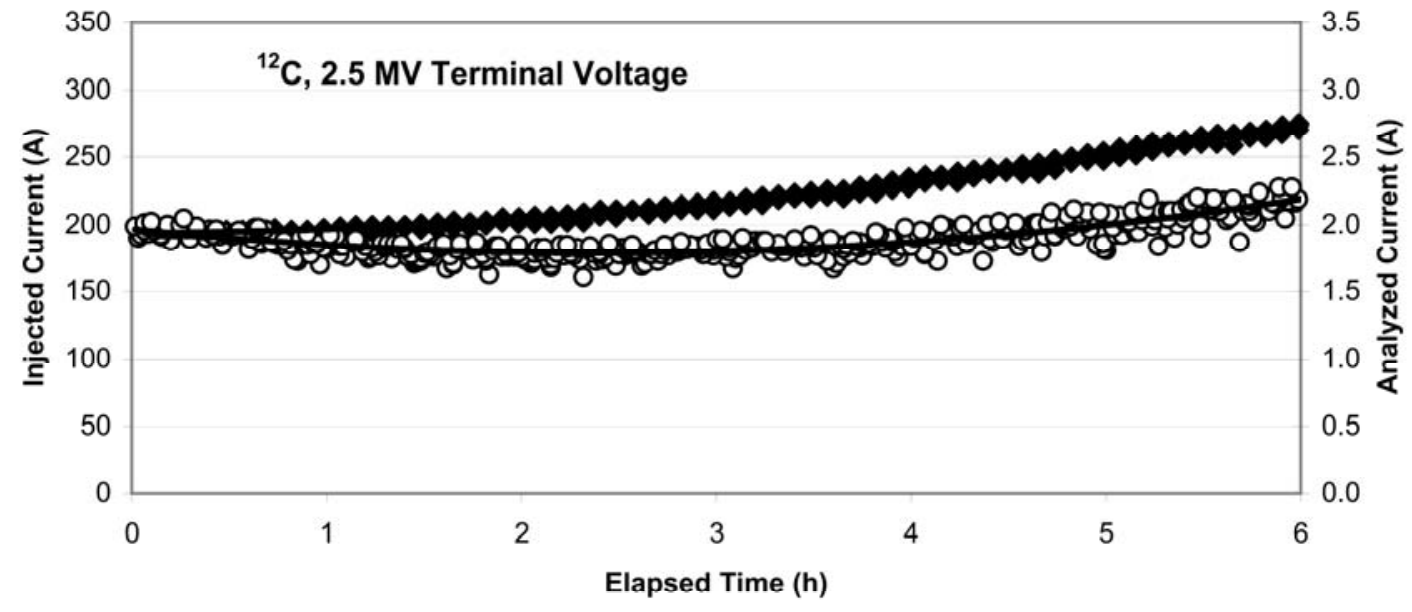

Figure 4

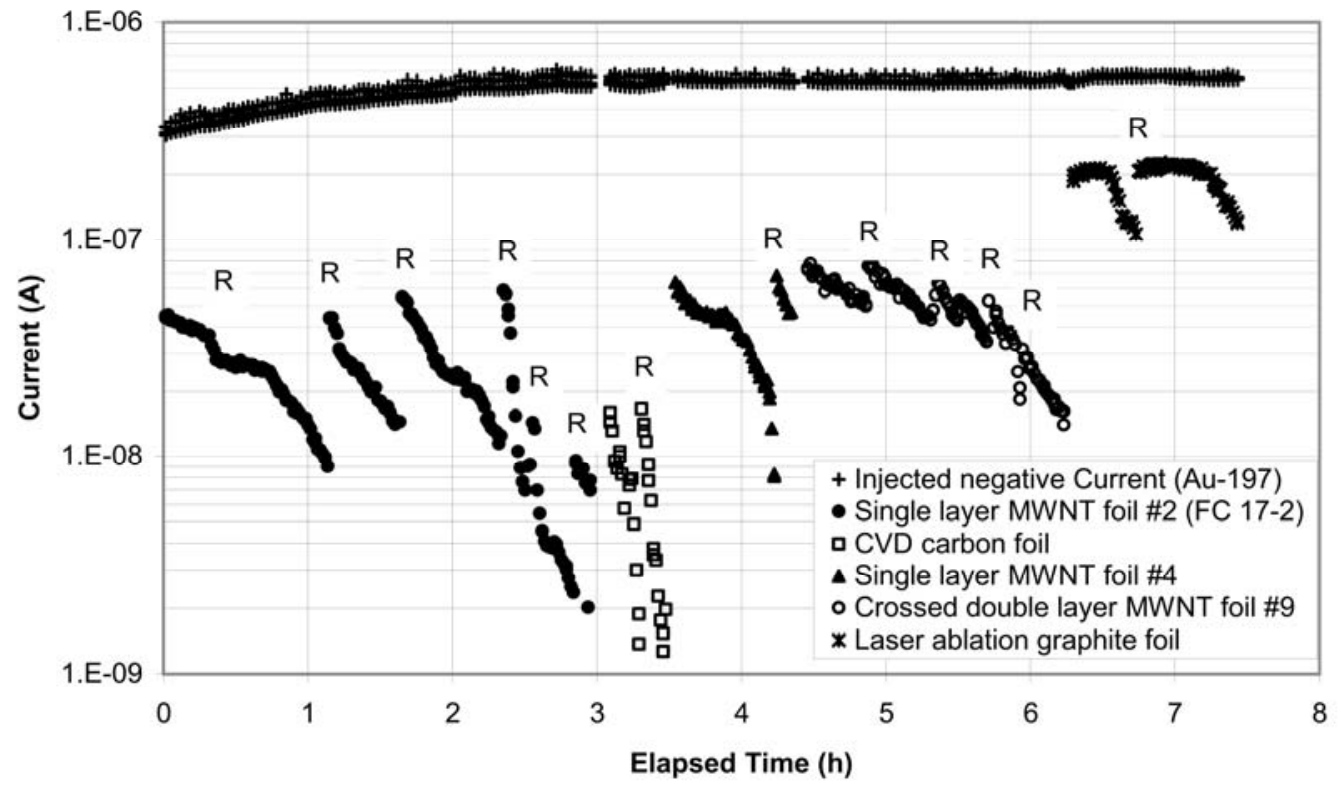

Figure 5 